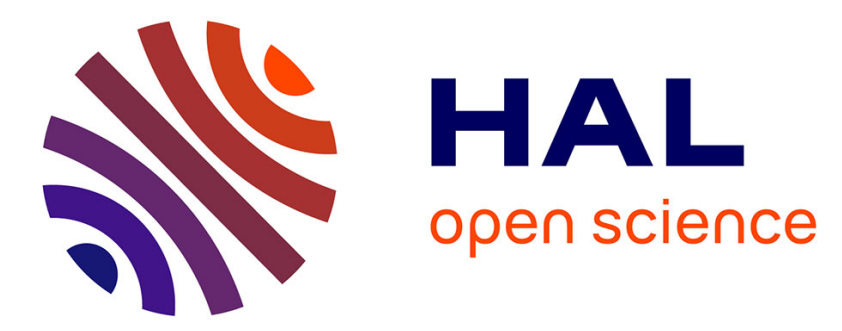

\title{
Dimensionless study of the total response of thermally stimulated luminescence
}

\author{
Franck Mady, Dominique Lapraz, Philibert Iacconi
}

\section{To cite this version:}

Franck Mady, Dominique Lapraz, Philibert Iacconi. Dimensionless study of the total response of thermally stimulated luminescence. Radiation Measurements, 2008, 43 (2-6), pp.180-184. 10.1016/j.radmeas.2007.11.022 . hal-00437681

\section{HAL Id: hal-00437681 \\ https://hal.science/hal-00437681}

Submitted on 11 Feb 2010

HAL is a multi-disciplinary open access archive for the deposit and dissemination of scientific research documents, whether they are published or not. The documents may come from teaching and research institutions in France or abroad, or from public or private research centers.
L'archive ouverte pluridisciplinaire HAL, est destinée au dépôt et à la diffusion de documents scientifiques de niveau recherche, publiés ou non, émanant des établissements d'enseignement et de recherche français ou étrangers, des laboratoires publics ou privés. 


\title{
Dimensionless study of the total response of thermally stimulated luminescence
}

\author{
Franck Mady ${ }^{*}$, Dominique Lapraz and Philibert Iacconi \\ LPES-CRESA (EA 1174), University of Nice-Sophia Antipolis, Parc Valrose, Nice, France
}

Received 13 August 2007; received in revised form 13 August 2007; accepted 13 August 2007

\begin{abstract}
The response of thermally stimulated luminescence (TSL) is investigated theoretically. An original dimensionless treatment is proposed, first to highlight the key dimensionless factors rationalizing the competition between the mechanisms at play during the TSL readout, and second to clean up the approach from any particular values of the relevant physical parameters. The approach holds for an unlimited number of interactive traps. The total response from a current trap is derived and cast into simple formal expressions as a function of the recombination cross-section, the set of trapping cross-sections, and of initial trap occupancies (i.e. those existing before the readout of the current peak). This allows one to calculate each peak response by taking proper account of carrier transfers accompanying the successive peak readouts.
\end{abstract}

Keywords: TSL; Dose response;

\section{Introduction}

Thermally stimulated luminescence (TSL) is used as a routine solid-state dosimetry technique. Nevertheless, its modelling still lacks of dimensionless analysis highlighting the key factors which govern its response. Basic mechanisms at play - trapping, detrapping and recombination - are usually accounted for by a set of differential kinetic equations, but their resolution has not provided the sought factors: simplest formal treatments were based on severe approximations (wellknown kinetic order models with single active traps and quasiequilibrium approximation). More realistic models, involving several interactive traps, have been treated numerically for restricted sets of parameter values (see e.g. Chen et al., 1981; Bull et al., 1986; Berkane-Krachai et al., 2002; Mady et al., 2006), or formally with significant complexity (Lewandowski and McKeever, 1991).

In dosimetry, what is expected from a TSL theory is basically a relation between the absorbed dose and the measured response. The total response integrated over a relevant temperature range is only governed by intrinsic dosimeter properties, as trapping and recombination crosssections or trap concentrations, and by trap occupancy at the onset of this range. This occupancy depends on the radiation dose and dose-rate given to the dosimeter prior to the readout (Chen et al., 1981). Thus, the dose response modelling first requires the determination of the initial trap occupancy yielded by irradiation. It then demands to calculate the total response as a function of initial trap occupancy. The present work focuses on the latter stage.

\footnotetext{
${ }^{*}$ Corresponding author. Tel.: +33492076330; fax: 33492076336

E-mail adresses : mady@unice.fr (F. Mady),

lapraz@unice.fr (D. Lapraz), iacconi@unice.fr (P. Iacconi)
}

Since we deal with integrated responses, there is no point in considering a time or temperature-resolved approach here. This frees us from the mathematical complexity that forces to resort to numerical resolution and makes the theory independent of the nature or kinetics of the stimulation allowing the readout. Then, general expressions of the response are derived within a simple formalism. The key coefficients governing the response are naturally pointed out by the dimensionless treatment.

\section{Theoretical route of attack}

A typical system similar to that of usual TSL models is illustrated in Fig. 1. TSL is assumed to originate from the stimulated release of trapped electrons which subsequently recombine with holes on recombination centres (RC). The treatment being independent of the carrier polarity, it would hold for holes as well. A set of $M$ discrete gap levels is considered. Each level is denoted by its order index $k$ which increases with trap depth $(1 \leq k \leq M)$. $\beta_{k}$ is the trapping coefficient of traps $k$ (electron thermal velocity multiplied by the capture cross-section of traps $k$ ), while $\gamma$ is the recombination coefficient (electron thermal velocity multiplied by the recombination cross-section). $N_{k}$ and $n_{k}$ are the densities of traps $k$ and of electrons in traps $k$ respectively, and $h$ is the concentration of holes on RC. 


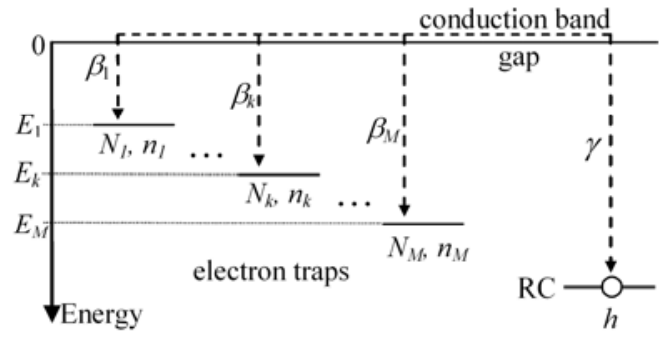

Fig 1. Typical energy scheme showing traps, recombination centres (RC), trapping and recombination transitions. Trap depths $E_{k}$ increase with $k$.

The total TSL response of the $p^{\text {th }}$ peak, due to the emptying of trap $p$, results from a conversion between two equilibriums characterized by the absence of carriers in the conduction and valence bands. At initial state, electrons are distributed on traps $p$ to $M$, levels 1 to $(p-1)$ having been emptied at lower temperature. At final state, electrons that have escaped recombination are retrapped on traps $(p+1)$ to $M$.

In what follows, initial concentrations are indicated by a superscript $(p)$. Final populations are denoted by a superscript $(p+1)$ since they also define initial occupancies relative to the emptying of the $(p+1)^{\text {th }}$ trap. The sample neutrality is then written as:

$h^{(p)}=\sum_{k=p}^{M} n_{k}^{(p)}$, or $h^{(p+1)}=\sum_{k=p+1}^{M} n_{k}^{(p+1)}$.

During the readout of the current peak $p, n_{k}$ (for $p+1 \leq k \leq M$ ) and $h$ obey usual equations, where $n_{\mathrm{c}}$ is the transient electron concentration in the conduction band:

$\frac{d n_{k}}{d t}=\beta_{k} n_{c}\left(N_{k}-n_{k}\right)$

$\frac{d h}{d t}=-\gamma n_{c} h$.

For levels $p+1 \leq k \leq M$, thermal release is neglected within the temperature range of the $p^{\text {th }}$ peak. Then, partial integration of Eq. (2) and (3) between initial and final states gives:

$n_{k}^{(p+1)}-n_{k}^{(p)}=\left(N_{k}-n_{k}^{(p)}\right) \times\left[1-\left(\frac{h^{(p+1)}}{h^{(p)}}\right)^{\frac{\beta_{k}}{\gamma}}\right], k \geq p+1$.

\section{Dimensionless formulation and total response}

After Eq. (4), competition between recombination with holes on $\mathrm{RC}$ and retrapping on levels $k>p$ is naturally rationalized by the $\alpha_{k}=\beta_{k} / \gamma$ ratios. We furthermore define trap occupancies $\varphi_{k}$ as the fraction of occupied traps $n_{k} / N_{k}$, and introduce the proportion $\xi_{k}$ of traps $k$ with respect to the total density of traps: $\xi_{k}=N_{k} / \sum_{j=1}^{M} N_{j}$.

The total TSL response of the $p^{\text {th }}$ peak is equal to the total number of recombination taking place throughout the peak readout. If every recombination is radiative, this response is equal to $h^{(p)}-h^{(p+1)}$, the variation of the hole concentration on RC between initial and final states. We then define the dimensionless total response $R_{p}$ of the $p^{\text {th }}$ peak by:
$R_{p}=\frac{h^{(p)}-h^{(p+1)}}{N_{p}}$.

The normalization is such that $0 \leq R_{p} \leq 1$. The case $R_{p}=1$ is achieved when all traps $k \geq p$ are initially saturated.

From (1), (4) and (5), one obtains the formal equation of $R_{p}$ for initial occupancy rates $\varphi_{k}^{(p)}$, trap weights $\xi_{k}$ and crosssection ratios $\alpha_{k}(k=p . . M)$ :

$R_{p}=\varphi_{p}^{(p)}-\frac{1}{\xi_{p}} \sum_{k=p+1}^{M} \xi_{k}\left(1-\varphi_{k}^{(p)}\right)\left[1-\left(1-\frac{\xi_{p} R_{p}}{\sum_{k=p}^{M} \xi_{k} \varphi_{k}^{(p)}}\right)^{\alpha_{k}}\right]$.

This leads to $R_{p}=\varphi_{p}{ }^{(p)}$, that is $h^{(p)}-h^{(p+1)}=n_{p}{ }^{(p)}$, in the absence of traps deeper than the $p^{\text {th }}$ one $\left(\xi_{k}=0\right.$ for $\left.k>p\right)$, or if deep traps are initially saturated $\left(\varphi_{k}{ }^{(p)}=1\right.$ for $\left.k>p\right)$. Then, carriers released from the $p^{\text {th }}$ trap can not be retrapped (except on the $p^{\text {th }}$ trap itself from which they are rapidly re-emitted). Finally, they all recombine and take part in the total response.

\section{Case of a single trapping cross-section}

When traps have a same trapping cross-section, i.e. $\alpha_{k}=\alpha$ for $k=1 . . M$, Eq. (4) shows that $\left(\varphi_{k}^{(p+1)}-\varphi_{k}^{(p)}\right) /(1-$ $\left.\varphi_{k}^{(p)}\right)$ does no depend on $k . \varphi_{k}^{(p+1)}$ is therefore independent of $k$ if $\varphi_{k}{ }^{(p)}$ is the same for any $k$. This is actually true regardless of initial and final moments, even between $\varphi_{k}{ }^{(1)}$ and trap occupancy before irradiation: if traps are all initially empty, they will fill up parallel to one another under irradiation. Thus, the $\varphi_{k}^{(1)}$ and the subsequent $\varphi_{k}^{(p)}$ for $p>1$ will be all independent of $k$ and Eq. (6) will reduce to:

$R_{p}=\varphi^{(p)}-\frac{\xi_{D}^{(p)}}{\xi_{p}}\left(1-\varphi^{(p)}\right)\left[1-\left(1-\frac{\xi_{p} R_{p}}{\varphi^{(p)}\left(\xi_{p}+\xi_{D}^{(p)}\right)}\right)^{\alpha}\right]$

Now, $\varphi^{(p)}$ is the trap occupancy of traps $k \geq p$ before the $p^{\text {th }}$ peak readout and $\xi_{D}^{(p)}$ is the fraction of traps deeper than the $p^{\text {th }}$ one, that is $\xi_{D}^{(p)}=\sum_{k=p+1}^{M} \xi_{k}$. Note that $\xi_{D}{ }^{(0)}=1$ and $\xi_{D}{ }^{(M)}=0$.

Fig. 2 and 3 show the $R_{2}$ plots obtained after Eq. (7) as a function of the initial occupancy $\varphi^{(2)}$. These examples consider a three trap system $(M=3)$ with $\xi_{1}=20 \%, \xi_{2}=30 \%$, $\xi_{3}=50 \%$ (Fig. 2) and $\xi_{1}=69 \%, \xi_{2}=30 \%, \xi_{3}=1 \%$ (Fig. 3). They well illustrate the effect of the deep trap density $\left(N_{3}\right.$ here) on the total response. 


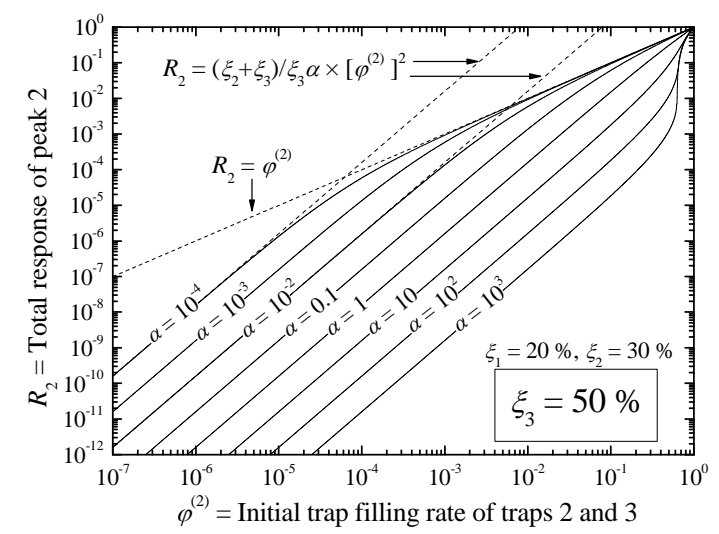

Fig 2. Dimensionless integrated response of peak 2 as a function of initial occupancy of traps 2 and 3 for a three trap system with $\xi_{1}=20 \%, \xi_{2}=30 \%$ and $\xi_{3}=50 \%$. The three traps have the same trapping cross-section ( $\alpha$ values are indicated on the plots).

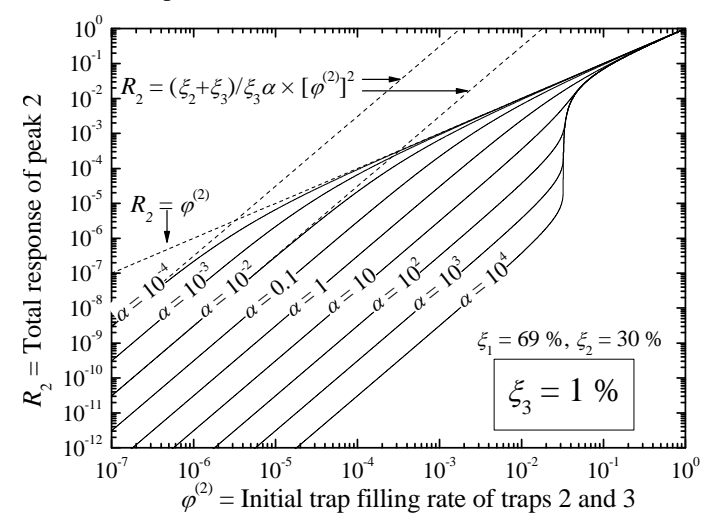

Fig 3. Same as Fig. 2 for $\xi_{1}=69 \%, \xi_{2}=30 \%$ and $\xi_{3}=1 \%$. Note the difference due to the concentration of the deepest (third) trap at high $\alpha$ and high initial occupancy rate.

\section{Useful approximations}

Explicit approximations of Eq. (6) can be derived for convenience. Let us first examine the strong retrapping hypothesis, when most carriers released from the $p^{\text {th }}$ trap are captured on levels $(p+1)$ to $M$ instead of recombining. Then, $\left(h^{(p)}-h^{(p+1)}\right) / h^{(p)}<<1$, that is $\xi_{p} R_{p}<<\sum_{k=p}^{M} \xi_{k} \varphi_{k}^{(p)}$, and a first order expansion of (6) readily gives:

$$
R_{p}=\frac{\varphi_{p}^{(p)}}{1+\frac{\sum_{k=p+1}^{M} \alpha_{k} \xi_{k}\left(1-\varphi_{k}^{(p)}\right)}{\sum_{k=p}^{M} \xi_{k} \varphi_{k}^{(p)}}}
$$

In case of weak retrapping, carriers released from the $p^{\text {th }}$ trap mostly recombine with holes and $1-R_{p} / \varphi_{p}{ }^{p)}<<1$. If one further assumes that negligible retrapping is due to the predominance of the recombination cross-section over every trapping one rather than to saturation of traps $k>p$, one also has $\alpha_{k}<<1$ for $k=p+1 . . M$. Then, the approximated $R_{p}$ obtained by first order expansions of Eq. (6), is found to be the same as Eq. (8). Thus, Eq. (8) seems to be an appropriate approximation in either limit situation.

If a single trapping cross-section is assumed $\left(\alpha_{k}=\alpha\right)$, Eq. (8) can be written as:

$$
R_{p}=\frac{\varphi^{(p)}}{1+\alpha \frac{\xi_{D}^{(p)}}{\xi_{D}^{(p-1)}} \times \frac{1-\varphi^{(p)}}{\varphi^{(p)}}}
$$

At weak trap occupancy, the second term in the denominator of Eq. (9) is much greater than unity and $R_{p}$ obeys:

$R_{p}=\frac{\xi_{D}^{(p-1)}}{\alpha \xi_{D}^{(p)}} \varphi^{(p)^{2}}$.

The response is then quadratic with respect to initial occupancy (see examples of Fig. 2 and 3). As $\varphi^{(p)}$ increases, the denominator of Eq. (9) tends to unity and the response becomes linear, independent of $\alpha: R_{p} \sim \varphi^{(p)}$. Such quadratic and linear TSL responses have already been predicted (Sunta et al., 1994; Kristianpoller et al., 1974, Mady et al., 2006). They are formally demonstrated here, and quantified as a function of the physical parameters of the standard model.

Eq. (9) becomes exact, i.e. rigorously equivalent to Eq. (7), when $\alpha=1$. For examples of Fig. 2 and 3, Eq. (9) slightly overestimates $R_{p}$ for $\alpha<1$ and yields an underestimated value for $\alpha>1$. The relative error is only about a few percent over a wide range of initial occupancy, but it forms a peak around the quadratic-to-linear transition. The peak maximum is at 5$10 \%$ for $\alpha<1$, so the approximation is always reliable in this case. This error is much greater for $\alpha>>1$, when the transition appears as a 'vertical' increase (e.g. when $\varphi^{(2)}$ is in the range $0.5-1$ in Fig. 2 or $0.01-0.1$ in Fig. 3). Then, the term in square brackets of Eq. (7) tends to unity and a more adequate approximation is:

$R_{p}=\left(1+\frac{\xi_{D}^{(p)}}{\xi_{p}}\right) \varphi^{(p)}-\frac{\xi_{D}^{(p)}}{\xi_{p}}$.

This approximation also corresponds to a linear increase of $R_{p}$, independent of $\alpha$. It tends towards $R_{p} \sim \varphi^{(p)}$ as $\varphi^{(p)}$ approaches unity. A detailed examination of Eq. (7) indicates that Eq. (11) is valid when $\varphi^{(p)}>\varphi_{c}$. The critical value $\varphi_{c}$ is $\xi_{D}^{(p)} / \xi_{D}^{(p-1)}$; it gives the location of the vertical transition: $\varphi_{c}=0.625$ in Fig. 2 and $\varphi_{c}=3.2 \times 10^{-2}$ in Fig. 3. At $\varphi<<\varphi_{c}$, the quadratic regime is accurately described by Eq. (9) or (10), as for $\alpha<1$.

In Eq. (8), for traps with different trapping cross-sections and initial occupancies, it is much more difficult to highlight linear and quadratic regimes of $R_{p}$ according to $\varphi_{p}{ }^{(p)}$. However, taking $\alpha_{k}>>1$ for $k=p+1 . . M$ in Eq. (6) still yields a linear relation at high occupancies:

$$
R_{p}=\varphi_{p}^{(p)}+\frac{1}{\xi_{p}} \sum_{k=p+1}^{M} \xi_{k} \varphi_{k}^{(p)}-\frac{\xi_{D}^{(p)}}{\xi_{p}} \text { if } \sum_{k=p}^{M} \xi_{k} \varphi_{k}^{(p)}>\xi_{D}^{(p)}
$$

\section{Iterative calculation of successive peak responses}

A simple iterative scheme can be proposed to predict the total response of the successive glow peaks. First, the total response $R_{p}$ of a current peak $p$ is given by Eq. (6). Second, Eq. (4) allows the calculation of initial trap occupancies $\varphi_{k}^{(p+1)}$ at the $(p+1)^{\text {th }}$ step as a function of initial occupancies at the $p^{\text {th }}$ step and of the $p^{\text {th }}$ peak response. For $p+1 \leq k \leq M$ : 
$\varphi_{k}^{(p+1)}=\varphi_{k}^{(p)}+\left(1-\varphi_{k}^{(p)}\right) \times\left[1-\left(1-\frac{\xi_{p} R_{p}}{\sum_{m=p}^{M} \xi_{m} \varphi_{m}^{(p)}}\right)^{\alpha_{k}}\right]$

If initial occupancies are known at the $p^{\text {th }}$ step, $R_{p}$ can be calculated either numerically by (6) or analytically with the approximated form (8) if applicable. Eq. (13) then provides new occupancies at the $(p+1)^{\text {th }}$ step, thus enabling the calculation of $R_{p+1} \ldots$ The problem now lies in finding the first trap filling state $\varphi_{k}{ }^{(1)}$ left by irradiation in the dosimeter. This state depends on the material, on the absorbed dose and on the dose-rate. Its general calculation is worth peculiar publication and is far beyond the scope of this work. The following section just presents a particular example where radiationinduced occupancies and the related responses can be calculated formally as a function of the absorbed dose.

\section{Application example}

The case $\alpha_{k}=\alpha=1$, corresponding to a single crosssection equal to the recombination one, ensures a full formal treatment. Then, trap occupancies are independent of the trap level $k$ at any step $p$ and Eq. (9) and (13) leads to:

$\varphi^{(p+1)}=\frac{\xi_{D}^{(p-1)} \varphi^{(p)}}{\xi_{p} \varphi^{(p)}+\xi_{D}^{(p-1)}}$.

For the sake of example, the assumption of low doses is made here: the dimensionless dose coefficient $D$, defined as the ratio of the total density of electron-hole pairs excited by irradiation to the total concentration of traps (sum of the $N_{k}$ for $k=1$.. $M$ ), is such that $D<<1$. Then, initial trap filling is approximately $\varphi^{(1)}=D$ regardless of the dose-rate and the following equations can be derived from Eq. (14) and (9):

$$
\begin{aligned}
& \varphi^{(p)}=\frac{D}{D+\xi_{D}^{(p-1)}(1-D)}, \\
& R_{p}=\frac{D^{2}}{\left(D+\xi_{D}^{(p-1)}\right)\left(D+\xi_{D}^{(p)}\right)} .
\end{aligned}
$$

The TSL response of the $p^{\text {th }}$ peak depends on the fractions of traps deeper than the $(p-1)^{\text {th }}$ and $p^{\text {th }}$ ones. If these fractions were very small, such as $\xi_{D}^{(p-1)}, \xi_{D}^{(p)}<<D<<1, R_{p}$ would tend to unity because of trap saturation. However, $\xi_{D}{ }^{(p-1)}$ and $\xi_{D}{ }^{(p)}$ are likely to be much greater than $D$ if $D<<1$, so $R_{p}$ is expected to be proportional to $D^{2}$. This quadratic dose response holds for all peaks $p$, except for $p=M$ since $\xi_{D}{ }^{(M)}=0$. The response of this last peak varies linearly with the dose because of the absence of deeper traps.

\section{Conclusion}

Dimensionless expressions of the total TSL response have been derived in the context of a simple model accounting for essential physics in a set of interactive traps. The obtained equations clearly demonstrate that the $\alpha_{k}=\beta_{k} / \gamma$ ratios are the only relevant coefficients that determine the effect of trapping and recombination cross-sections on each peak response. This fact, which has already been observed from simulations (Mady et al, 2006), is here formally demonstrated.

Starting from a given dosimeter model - defined by its cross-section ratios $\alpha_{k}=\beta_{k} / \gamma$ and trap weights $\xi_{k}$ - and from a set of initial trap occupancies, the iterative procedure of section 6 gives the response of successive peaks associated with traps of increasing depths. Carrier transfer, i.e. enhancement of deep trap occupancies due to retrapping of carriers released during the readout of a shallow level, is naturally taken into account. The main assumption of the treatment is the absence of detrapping from traps deeper than the active one in the temperature range of the current peak. Therefore, what we refer to as successive peaks must be separate peaks. If a group of several neighbouring traps yields overlapping peaks, the formalism will provide the total response of the peak group if all associated traps have the same $\beta$. Then, subscripts $p$ or $k$ refer to the whole group of traps rather than to a single level.

Apart from the particular case $\alpha=1$ where explicit equations and formal solutions exist, the proposed procedure can be implemented in a computer program to perform very efficient calculations of the dose and dose-rate response of any peak of a series. This requires the calculation of initial trap occupancies as a function of dose and dose-rate. We currently consider this issue with an adequate dimensionless formalism which is to be presented in a forthcoming publication.

\section{References}

Berkane-Krachai, A., Iacconi, P., Bindi, R., Vinceller, S., 2002. Analysis with a multilayer model of heating rate effect on thermally stimulated luminescence, conductivity and exoelectronic emission of $\alpha-\mathrm{Al}_{2} \mathrm{O}_{3}$. J. Phys. D: Appl. Phys. 35, pp 1895-1902.

Bull, R.K., McKeever, S.W.S., Chen, R., Mathur V.K., Rhodes, J.F., Brown, M.D., 1986. Thermoluminescence kinetics for multipeak glow curves produced by the release of electrons and holes. J. Phys. D: Appl. Phys. 19, pp 13211334.

Chen, R., McKeever, S.W.S., Durrani, S.A., 1981. Solution of the kinetic equations governing trap filling. Consequences concerning dose dependence and dose-rate effects. Phys. Rev. B 24 (9), pp 4931-4944.

Kristianpoller, N., Chen, R., Israeli, M., 1974. Dose dependence of thermoluminescence peaks. J. Phys. D: Appl. Phys. 7, pp 1063-1072.

Lewandowski, A.C., McKeever, S.W.S., 1991. Generalized description of thermally stimulated processes without the quasiequilibrium approximation. Phys. Rev. B 43 (10), pp 8163-8178.

Mady, F., Bindi, R., Iacconi, P., Wrobel, F., 2006. Influence of absorbed dose and deep traps on thermoluminescence response: a numerical simulation. Radiat. Prot. Dosim. 119 (1-4), pp 37-40.

Sunta, C.M., Yoshimura, E.M., Okuno, E., 1994. Supralinearity and sensitization of thermoluminescence. Part I: a theoretical treatment based on an interactive trap system. J. Phys. D: Appl. Phys. 27, pp 852-86. 